\title{
Period determinations of spotted stars
}

\author{
D. Clarke ${ }^{\star}$ \\ Department of Physics and Astronomy, University of Glasgow, Glasgow, G12 8QQ, Scotland, UK \\ Received 17 December 2002 / Accepted 9 June 2003

\begin{abstract}
The general behaviours of photometric, radial velocity and polarimetric observational investigations are explored in terms of the signals generated by a spot or patch in co-rotation with a star. It is demonstrated that the results of any period analysis of data depends on the nature of the chosen measurement diagnostic. Unlike photometry, analysis of radial velocity measurements is likely to provide considerable power at periods corresponding to the harmonics of the stellar rotational period. This conclusion may have bearing on Harmanec's "submultiple period scenario" in connection with the understanding of the behaviour of early-type stars. Period analysis of polarimetric data is also likely to provide strong power in the harmonics of the fundamental rotational period. This analysis of the signals from the three basic observational diagnostics shows the dangers of simply using one measurement parameter alone to investigate stellar temporal behaviour and how interpretational confusion can arise when the results from different exercises are compared.
\end{abstract}

Key words. stars: early-type - stars: oscillations - stars: rotation

\section{Introduction}

In a recent paper, Harmanec (1999) has continued the discussion on the "understanding of rapid line-profile and light variations of early-type stars". At issue is the interpretation of periodicity detections associated with photometry, with radial velocities variations and with the temporal progression of features across line profiles. The chief concern relates to models based either on structures co-rotating with the star or on nonradial pulsations, these two possible underlying mechanisms for the cyclical variability being in competition for acceptance. The problem has been widely aired in the literature over the years. Although Harmanec very openly leans to interpretations that the generated signatures arise from surface phenomena rotating with the star or to co-rotating circumstellar clouds, the various arguments of the two camps have been succinctly summarised by him (see Harmanec 1989, 1999; Harmanec \& Tarasov 1990). Observational material, schemes of the various kinds of parametric data reduction with their associated problems and the astrophysical arguments have been carefully treated in discussions by him.

In covering the development of the accruing data and the way in which the arguments have swung backwards and forwards, observations of two stars, $\epsilon \operatorname{Per}(=\mathrm{HD} 24760$ ) and $\zeta \mathrm{Oph}(=\mathrm{HD} 149757)$ were highlighted in the third of Harmanec's papers (see Harmanec 1999). In this work, the underlying theme of the discussion relates to the fact that many of the reported periods from different observational studies appear to be integer submultiples of some longer period.

\footnotetext{
* e-mail: d.clarke@astro.gla.ac.uk
}

For the case of $\epsilon$ Per, a summary of periods determined from various kinds of measurement analysis by different observers is provided by Harmanec (1999) - his Table 1. The cited results include values of periods determined from photometry at specific wavelength positions within line profiles, particularly at the line centre, e.g., see Gies \& Kullavanijaya (1988) and from the fitting of theoretical models related to the effects of non-radial pulsations on the behaviour of line profiles, e.g., see Smith (1985). The list is set in decreasing value of the detected period linked to a matching integer submultiple $(N)$ of a single (photometric) period $\sim 1.12$ with periods being referenced at values of $N=2,5,6,7,8,9,12$, and 14 . Although not mentioned specifically in Harmanec's table, in addition to determining periods by model fitting with respect to non-radial pulsations, one of the cited papers (Smith et al. 1987) includes analysis of broadband photometry, with the determination of a period corresponding to $N=7$. The relationships of these determined periods to a basic period is a development of an earlier report by Harmanec (1987) in which the submultiple scenario was first noted for $\epsilon$ Per, with a similar thesis being promoted for the Be star, $\mu$ Cen (=HD 120324). It may be noted that the description of there being periodic signatures associated with $\mu$ Cen which are simply submultiples of an underlying fundamental period now appears to be too simplistic; according to a radial velocity analysis of this star, Rivinius et al. (1998) advocate the presence of six different periods which are not exact submultiples of a single, longer period variation. These, findings, however, do not stall the general theme explored here.

The aim of this paper is to demonstrate that the outcome of any simple, non-parametric, period analysis as applied to data 
depends on the type of diagnostic measurement used in relation to the fundamental cause of the variability. The explored example simply involves the presence of some physical feature within the photosphere such as a spot, co-rotating with the star, using the notion that the disturbance affects measurements of photometry, radial velocity and polarization according to the rotational phase. Although the study may have bearing on the argument associated with the origin of the various periodic signals determined from various measurements of early type stars, its purpose is more general to the analysis of any type of star exhibiting surface patches or circumstellar clouds giving rise to rotationally modulated signals. When such a disturbing feature appears on the limb of a star and moves across the projected stellar disk, its effect provides very different signals according to the observational diagnostic. For example, the photometric signal shows gradual changes at the appearance of the patch on the limb and with its progress across the face of the star, whereas the simultaneous engendered polarimetric signal is more likely to be sharper in its form. Thus Fourier analyses of the two kinds of signal will produce different results; most power will appear at low frequencies (longer periods) for photometry and at higher frequencies (shorter periods) for polarimetry, simply as a result of the natures of the differing types of signal, although they are generated by the same astrophysical cause.

The outcomes of the exercise suggest that in the case of the studies of early-type stars as outlined above, the integer submultiple period scenario may be reconciled in terms of features, patches or spots that co-rotate with the star. The plethora of reported period values may simply ensue from the differing fundamental forms of the underlying signals associated with the kind of applied observational diagnostic and data analysis procedure. Comparisons are made between the signals associated with photometry, spectral line photometry and radial velocity $(R V)$ measurements, all these being used in various observational studies. In addition, the exercise has been extended to demonstrate that if polarimetry were to be included as an observational diagnostic, analysis of these measurements is likely to provide harmonics of the rotational period with strengths differing from those obtained from photometry and spectroscopy.

Although the analysis in the paper is couched in terms of a "spot" model, the study has wider implications to other stellar investigations and demonstrates generally that results of period analyses "may appear" to be very different according to whether the data relate to the broad disciplines of photometry, spectroscopy or polarimetry but with the signal variations originating from the same astrophysical cause.

\section{The basic model}

Without invoking any astrophysical mechanisms related to the production of the localized disturbance, consider a point-like patch, co-rotating with the star, affecting the total radiation received from the object. The patch may be in the form of an active zone giving rise to a brightness enhancement or a cooler spot with depressed brightness, or some packet of material which absorbs or scatters radiation from deeper within the star. For the purpose of this paper, the exercise is promoted by

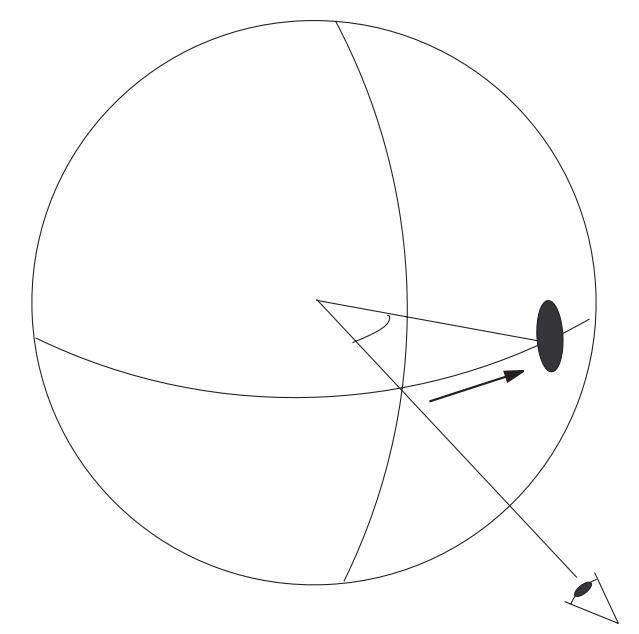

Fig. 1. The simple spot model displaying a feature on the stellar equator at an angle $\theta$ relative to the centre longitude; the axis of rotation of the star is normal to the line of sight with $\theta$ increasing as the feature co-rotates with the star with apparent movement from left to right.

considering the simplest of geometries such that the patch lies on the equator of the star and that the stellar inclination is $90^{\circ}$. For this scenario, the effects of the patch are apparent for half of the rotational period only as it traverses the projected stellar disk; during the other half of the period, the disturbance is blocked from view.

In the exercises below, the Fourier decomposition of any signal produced by the stellar model is based on the assumption that the modulation is recorded as a continuous function. In observational practice, the stellar variations would be sampled at discrete but arbitrary phase values and, in order to detect if periodicity were present, the record would be subject to some kind of period spectral analysis. Thus any periodogram is likely to exhibit features at positions corresponding to the Fourier components of the underlying signal, with the effects of noise, windowing and aliasing being superposed, these modifying the relative strengths of the detected harmonics. To a substantial degree, the window function problems may be reduced by using algorithms such as CLEAN (see Roberts et al. 1987), such treatment usually being applied.

\section{Photometric periodicities}

\subsection{Broadband photometry}

For the development of the thesis, consider the disturbance as a dark patch or spot, emitting less radiation per unit area than the undisturbed photosphere, its apparent position being defined by an angle $\theta$, this being zero when its projection is at the centre of the observed stellar disk (see Fig. 1). Suppose the patch, with specific brightness $B_{\mathrm{s}}$, occupies a fractional area, $f$, on the stellar surface which has an undisturbed specific brightness, $B_{\mathrm{p}}$. When the feature is apparent on the hemisphere facing the observer, the photometric signal and its variation may be written as

$I(\theta)=K\left[B_{\mathrm{p}}+f\left(B_{\mathrm{s}}-B_{\mathrm{p}}\right) \cos \theta\right]$, 


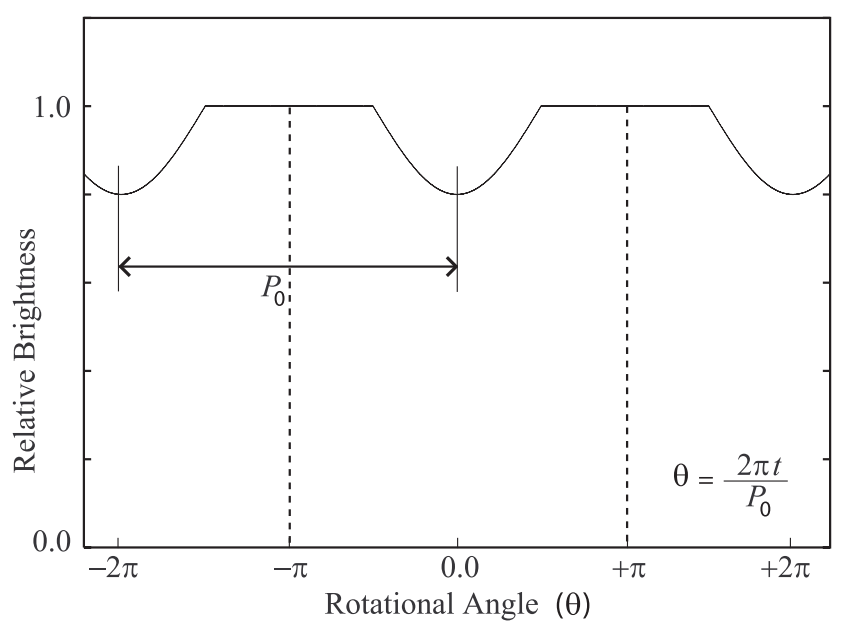

Fig. 2. The schematic behaviour of the relative brightness of a star based on the model as depicted in Fig. 1, with the amplitude, $A$, arbitrarily chosen as 0.2 . The horizontal axis $(\theta)$ is directly related to time by $\theta=2 \pi t / P_{0}$, where $P_{0}$ is the stellar rotational period. For the purpose of determining the coefficients of the Fourier series describing the waveform, the range of $\theta$ covering the period, $P_{0}$, was taken from $-\pi$ to $+\pi$.

where the $\cos \theta$ term describes the foreshortening of the projected area of the patch according to the angle $\theta$; the constant $K$ depends on the size of the star and on its distance from the Earth. When the feature is on the obscured hemisphere, the star displays a constant apparent brightness of $I_{0}=K B_{\mathrm{p}}$. Hence relative to the undisturbed level, the signal variation when the feature is apparent may be expressed as

$I(\theta) / I_{0}=1+A \cos \theta$,

where $A=f\left(B_{\mathrm{s}}-B_{\mathrm{p}}\right) / B_{\mathrm{p}}$. If photometry were to be continuously effected, a light-curve of the form as depicted in Fig. 2 would ensue. With $B_{\mathrm{s}}$ being less than $B_{\mathrm{p}}$, the perceived stellar brightness will fall as the patch appears on the approaching limb $(\theta=-\pi / 2)$, a minimum occurring when the spot is on the meridian $\left(\theta=0^{\circ}\right)$, and then will recover to the undisturbed brightness as it disappears at the receding limb $(\theta=+\pi / 2)$. Between the values of $\theta=-\pi$ and $-\pi / 2$, the star is seen in an undisturbed state, this also being the case for $\theta=+\pi / 2$ to $+\pi$.

In summary, the variation over one period may be expressed as

$I(\theta) / I_{0}=1+A \cos \theta:-\pi / 2 \leq \theta \leq+\pi / 2$

$I(\theta) / I_{0}=1:-\pi \leq \theta \leq-\pi / 2$ and $+\pi / 2 \leq \theta \leq+\pi$.

Analysis of Eqs. (3) in the form of a Fourier series shows that the oscillatory signal, with the period covering $-\pi$ to $+\pi$, may be represented by

$\frac{A}{2} \cos \theta+\frac{2 A}{\pi} \sum_{n=2}^{\infty}( \pm) \frac{\cos n \theta}{\left(n^{2}-1\right)}$,

the summation involving even harmonics only $(n=2,4,6, \ldots)$ and the sign within the summation being taken as positive when $n / 2$ is odd and negative when $n / 2$ is even. The expression demonstrates that there are significant contributions to the

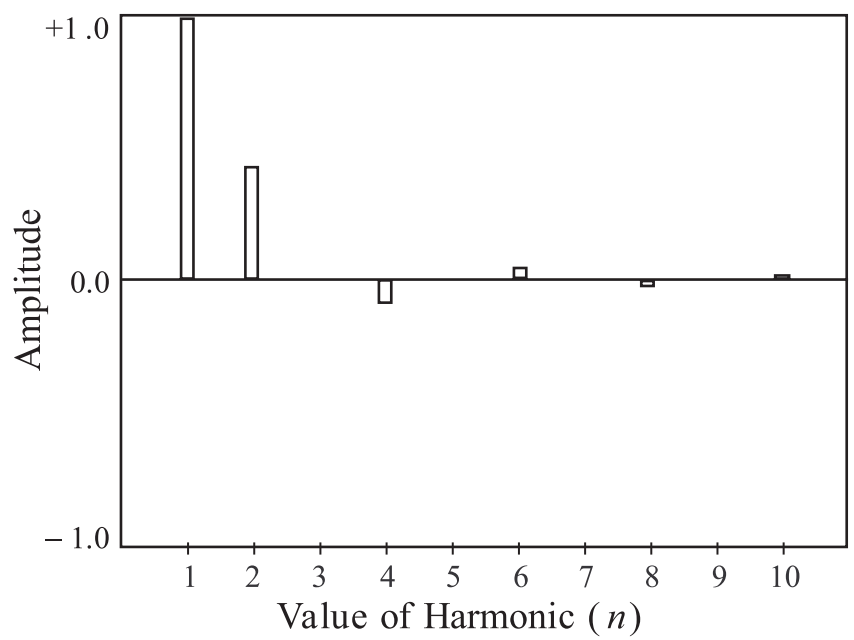

Fig. 3. The expected values of the amplitudes of the cosine components associated with the harmonics of the photometric signal as produced by a simple spot model. The amplitude values have been normalised to that of the first (fundamental) harmonic.

series from the first (rotational period) and second harmonic with the strengths of the even harmonics rapidly diminishing as the order increases, as depicted in Fig. 3.

By expressing the variation in terms of the rotational period of the star, $P_{0}$, the oscillatory component may be summarised by simply considering the contributions of the first and second harmonics. Thus

$I(t) \longrightarrow \frac{A}{2} \cos \frac{2 \pi t}{P_{0}}+\frac{2 A}{3 \pi} \cos 2 \frac{2 \pi t}{P_{0}}$

With respect to Eq. (5), it is noteworthy that in the photometric analysis of spotted K-type stars in the Pleiades cluster, Van Leeuwen et al. (1987) refined their period determinations by fitting a brightness variation based on the fundamental and second harmonic, this being justified simply on the notion that the procedure offered a very good fit to the light-curves.

For a more realistic scenario, the outcome of the analysis would be modified slightly as a result of the patch having finite size so that it would not appear and disappear instantly at the stellar edge; effects of limb darkening may also modify the basic "cosine" form of the brightness variation. The description of the light-curve would also require modification if the star's rotation axis were not at $90^{\circ}$ and if the patch had a latitude not equal to $0^{\circ}$. No matter the detail of geometry, to a first order, the brightness variation can be represented by the first two elements of the Fourier series.

It may also be noted that if the star carries more than one patch, the overall form of the modulation may be constructed simply by combining the various contributions. Any additional spot will carry the characteristic "cosine" form within the window between $+\pi$ and $-\pi$ but, as its position in the window will not be symmetric about $\theta=0$, both cosine and sine coefficients occur in its Fourier description. Rather than determining the form of the resulting modulation and determining its Fourier components, the power contained in the harmonics may be determined by performing the additions in Fourier space, essentially applying Parseval's theorem (e.g., see James 2002). 
The strength of each harmonic resulting from the effects of several spots is simply determined by addition of the component amplitudes of all the contributing cosine and sine terms, the amplitudes of these in turn being dependent on the spot strengths and on their locations along the stellar equator. In effect, this is likely to alter the relative strengths of the harmonics as displayed in Fig. 3. It may be noted also that the larger the number of spots, the smaller will be the final modulation relative to the undisturbed level of the signal, as the effects of each patch overlap and tend to cancel out. The outcome remains, however, with the likelihood that the first and second harmonics of the rotational frequency will dominate in the construction of the observed photometric waveform and of course in any periodogram of the measured variations.

\subsection{Line depth photometry}

In many spectroscopic studies, photometry is effected by measurements of line depths relative to the continuum. The exact expected behaviour of the associated waveform is very model dependent. To a first order, however, the fundamental behaviour may be represented as for photometry above but with an additional weighting function. For example, the contribution to the light level will be affected according to $\cos \theta$ as above but, at the time of the appearance of the spot on the limb, the rotational radial velocity concentrates any photometric change to the wing of the line rather than at its core. As the spot moves across the apparent disk of the star, the photometric changes affect the line core more and more. At this simplistic level, the overall modulation of the relative line depth might be expressed by applying a linear weighting function to Eq. (2) so that

$L(\theta) / L_{0}=1+B\left(1 \pm \frac{2 \theta}{\pi}\right) \cos \theta$,

where $L(\theta)$ is the line depth when the spot is placed at angle $\theta$ and $L_{0}$ is the undisturbed line depth when the spot is not apparent; $B$ relates the "strength" of the spot and the "+" sign is taken for $\theta \geq-\pi / 2 \leq 0$, with the "-" sign applied when $\theta \geq 0 \leq+\pi / 2$.

Fourier analysis of Eq. (6) provides a spectrum of harmonics (see Fig. 4) very different with respect to ordinary photometry. Although the first harmonic (fundamental) is dominant, both the second and third harmonics are strong, the third, in fact, being slightly stronger than the second. In contrast, the third harmonic in the simple photometric signal is completely absent.

If more than one disturbing patch is present, again by Parseval's theorem, the relative strengths of the various harmonics may be calculated according to the locations and strengths of the contributing spots. The likelihood, however, is that the dominant components in any simple Fourier analysis of line depth data are likely to comprise strong contributions from the first three harmonics.

It can be seen that there are already distinct outcomes in terms of the detection of periodicities between analyses of different kinds of photometric measurements. Similar differences are also apparent in respect of other observational diagnostics as expanded upon below.

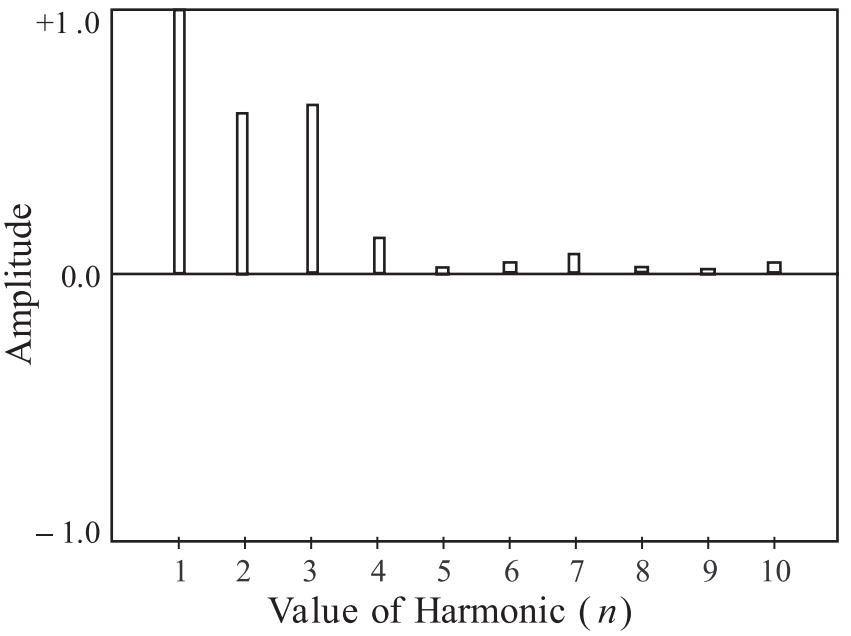

Fig. 4. The expected values of the amplitudes of the cosine components associated with the harmonics of the photometric signal based on measurements of the variation of line depths as produced by a simple spot model. The amplitude values have been normalised to that of the first (fundamental) harmonic.

\section{Periods based on RV determinations}

The effects of the patch or spot on its passage across the apparent stellar disk may also be monitored by high resolution spectrometry and the temporal changes of the line profile can be interpreted in terms of $R V$ variations. There are essentially two ways whereby this might be done.

The first involves measurement of the $R V$ associated with the complete recorded line profiles. The values may be determined by a variety of ways such as from calculations of the line centroid position, or from the turning point of a second order curve fitted to the line minimum, or by measuring the bisector velocities at a range of paired intensity levels on either side of the line profile minimum. Although the results of periodicity analysis is not mentioned in their paper, Harmanec \& Tarasov (1990) determined the behaviour of the centroid radial velocity of the He I line (6678 $\AA$ ) of $\epsilon$ Per, with the graphed time series displaying oscillatory modulation.

The second principle requires the identification of particular moving features, usually depressions, in the wings of lines and then monitoring their temporal progress across the parent line. There is a distinct advantage here in that the results may lead to the application of Doppler imaging techniques (see, for example, Vogt \& Penrod 1983). Again the graphs of the time series of the behaviour of "spectral blips" in $\epsilon$ Per has been presented by Harmanec \& Tarasov (1990).

If such accrued $R V$ data of either type are just simply analyzed for periodicity, the immediate apparent outcomes are likely to be different with respect to those resulting from photometry as described above. Also, the two outlined $R V$ techniques themselves are also likely to provide different outcomes because of the logistics associated with the measurement methods. Important issues are the underlying form of the basic signals and the way in which they are sampled. Consider in more detail the two types of $R V$ observation in turn. 


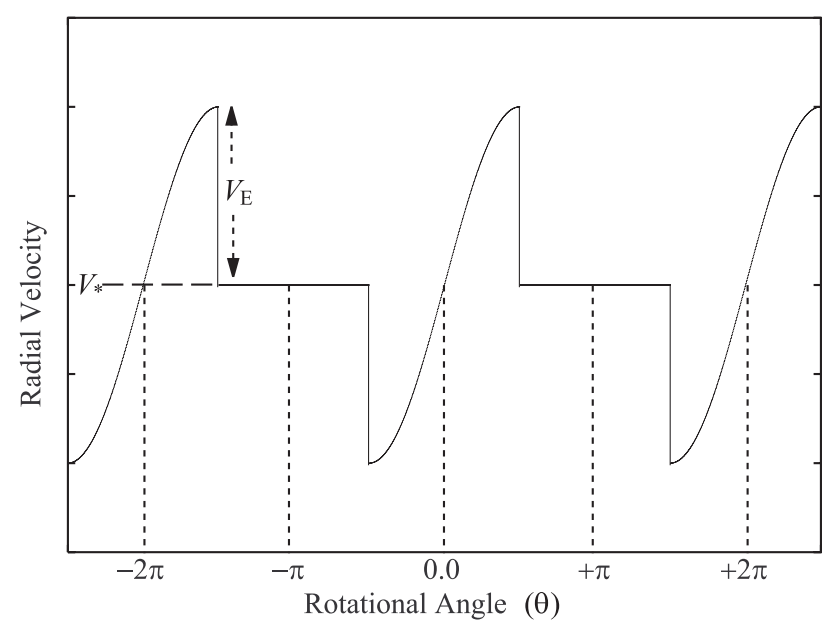

Fig. 5. The schematic behaviour of the radial velocity associated with a spot based on the model as depicted in Fig. 1. The horizontal axis $(\theta)$ is directly related to time by $\theta=2 \pi t / P_{0}$, the complete cycle covered by the interval $-\pi$ to $+\pi$.

\subsection{RV measurements of the whole line profile}

Again, using the model as depicted in Fig. 1, the $R V$ of the spectral feature produced by a spot relative to that of the undisturbed profile is simply $V_{\mathrm{E}} \sin \theta$, where $V_{\mathrm{E}}$ is the stellar equatorial velocity. The behaviour of its $R V$ value over the rotational period would follow a curve as illustrated in the central region of Fig. 5, between the limits $-\pi / 2$ to $+\pi / 2$. It will be appreciated immediately that because of the sudden sharp changes at $-\pi / 2$ and $+\pi / 2$, the Fourier series describing the complete waveform over the interval $-\pi$ to $+\pi$ will contain substantial strengths of higher harmonics of the basic period. This is demonstrated quantitatively by determining the Fourier series for the function summarised by

$R_{\mathrm{S}}=V_{*}+V_{\mathrm{E}} \sin \theta:-\pi / 2 \leq \theta \leq+\pi / 2$

$R_{\mathrm{S}}=V_{*}:-\pi \leq \theta \leq-\pi / 2$ and $+\pi / 2 \leq \theta \leq+\pi$,

where $V_{*}$ is the constant $R V$ of the star due to its space motion.

Analysis of Eq. (7) shows that the oscillatory signal, with the period covering $-\pi$ to $+\pi$, provides a series of the form

$\frac{V_{\mathrm{E}}}{2} \sin \theta+\frac{2 V_{\mathrm{E}}}{\pi} \sum_{n=2}^{\infty}( \pm) \frac{n \sin n \theta}{\left(n^{2}-1\right)}$,

the summation involving even harmonics only $(n=2,4,6, \ldots)$; the sign within the summation is taken as positive for $n=2$ and, for $n>2$, the sign is taken as positive when $n / 2$ is odd but negative when $n / 2$ is even. Comparison with Eq. (4) shows that the series involves sine functions rather than cosines, but comprises harmonics similar to the photometric case, except that the amplitude coefficients do not decay in the same way according to the order of the harmonic because of the factor of $n$ in the numerator.

In relation to the behaviour of real data, the simple model needs to be taken further. In estimating the effect that the patch has on the $R V$ determinations of the actual recorded lines, the strength and shape of its associated profile relative to that generated from the light of the undisturbed stellar disk needs to

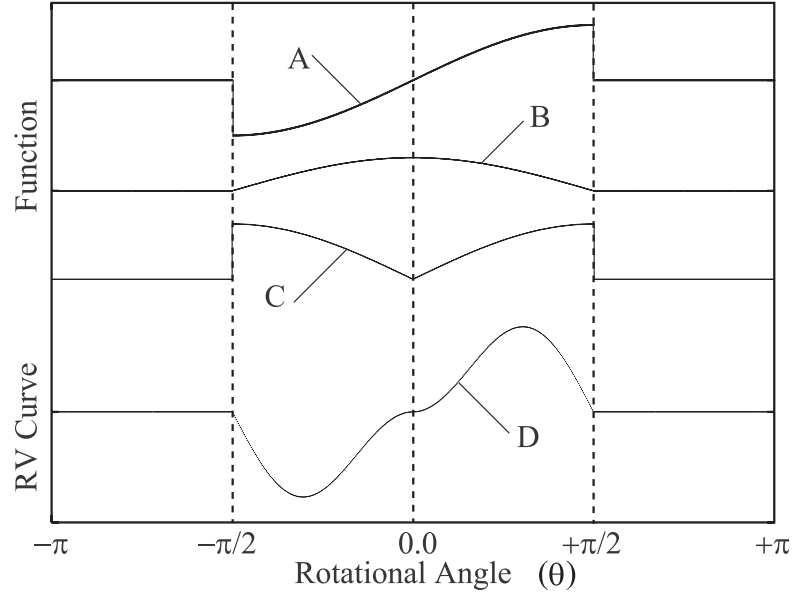

Fig. 6. The contributing functions involved in the development of the schematic behaviour (Curve D) of the $R V$ of a stellar line profile are depicted over a complete rotational interval of $\theta=-\pi$ to $+\pi$ (see Eq. (9)). The basic curve showing the $R V$ behaviour of the spectral feature associated with the disturbing patch is labelled as Curve A (see also Fig. 5). Curve B represents the cosine weighting factor to allow for foreshortening and Curve $\mathrm{C}$ represents the chosen weighting factor (| $\sin \theta \mid$ ) to account for the effect the disturbance has on the $R V$ of the overall stellar line. It can be seen from Curve D that the maximum measured $R V$ s occur when the patch is relative close to but not exactly on the stellar limb and that there is a flat section when the patch is located towards the centre of the projected stellar disk.

be known. To a first order, however, consider a model whereby the contribution of the patch simply depends on its $R V$ value relative to the undisturbed stellar line, with its effect scaled for foreshortening by a factor of $\cos \theta$, as for the case when determining its influence on the apparent brightness. As the final measured $R V$ value depends on the position of the disturbing effect within the parent line, a further weighting factor is required. Without modelling the situation in detail, it will be appreciated that the disturbance on the whole line will be greater when it is located in the high wing of the undisturbed line and will have zero effect when it coincides with its minimum. For simplicity in evaluating the Fourier series describing the $R V$ variation of the complete measured line, a weighting factor of $|\sin \theta|$ has been used. The effects of the various contributing functions are illustrated in Fig. 6 together with the expected typical $R V$ behaviour (Curve D) of the measured line. The outcome of this model shows that there is a rapid change in the measured $R V$ soon after the patch has appeared on the approaching limb; the $R V$ thereafter achieves a maximum before falling to a flat section when the patch is close to the centre of the projected stellar disk. After crossing the meridian, the $R V$ curve is mirrored but with opposite sense until the patch disappears at the receding limb.

For this simple scenario, the form of the $R V$ variations over the whole stellar rotational period may be summarised by
$R_{*}=V_{*}+V_{\mathrm{E}} \sin \theta \times \cos \theta \times|\sin \theta|: 0 \leq \theta \leq+\pi / 2$
$R_{*}=V_{*}:-\pi \leq \theta \leq-\pi / 2$ and $+\pi / 2 \leq \theta \leq+\pi$. 
Determination of the Fourier series shows that it comprises sine terms only with coefficients, $S_{n}$, given by

$$
\begin{aligned}
& S_{1}\left(P_{0}\right) \propto \frac{V_{\mathrm{E}}}{2 \pi}, \quad S_{2}\left(P_{0} / 2\right) \propto \frac{8 V_{\mathrm{E}}}{15 \pi}, \\
& S_{3}\left(P_{0} / 3\right) \propto \frac{V_{\mathrm{E}}}{6 \pi}, \quad S_{4}\left(P_{0} / 4\right) \propto-\frac{16 V_{\mathrm{E}}}{105 \pi}, \\
& S_{5}\left(P_{0} / 5\right) \propto-\frac{V_{\mathrm{E}}}{6 \pi}, \quad S_{6}\left(P_{0} / 6\right) \propto-\frac{8 V_{\mathrm{E}}}{315 \pi}, \\
& S_{7}\left(P_{0} / 7\right) \propto \frac{V_{\mathrm{E}}}{30 \pi}, \quad S_{8}\left(P_{0} / 8\right) \propto-\frac{32 V_{\mathrm{E}}}{3465 \pi}, \\
& S_{9}\left(P_{0} / 9\right) \propto-\frac{V_{\mathrm{E}}}{30 \pi}, \quad S_{10}\left(P_{0} / 10\right) \propto-\frac{40 V_{\mathrm{E}}}{1287 \pi}, \text { etc. }
\end{aligned}
$$

The values suggest that it is the second harmonic that carries the most power and its corresponding period is the most likely to show strongly in any periodogram. It can also be seen that there is significant power distributed among the higher harmonics, particularly the odd ones.

Further investigation of the model shows that the apparent $R V$ variation depends on the choice of weighting function associated with the effect that the patch has on the overall line profile according to the angle, $\theta$. For example, if the function is taken as $|\theta|$ rather than $|\sin \theta|$, this has the effect of moving the maximum and minimum $R V$ values to be closer to the times when the patch disappears or appears at the stellar limb $(\theta \sim+\pi / 2$ or $-\pi / 2$, respectively). In turn, this alters the distribution of power to the harmonics of the fundamental period. If the behavioural function for the $R V$ variation were to be derived more exactly, taking into account the shapes of the underlying undisturbed profile and the effect the disturbing patch induces according to $\theta$, the conclusion would be the same - higher harmonics are very likely to show in periodograms based on $R V$ measurements of complete stellar spectral lines.

Again, as for the case of photometric variations (Sect. 3), if several patches are present, their effect can be constructed by combining their signals according to the amplitudes and phases; the expected periodogram will again be of the form as depicted in Fig. 7. There will also be modifications to the $R V$ curve according to the physical size of the patches relative to the stellar radius, but the general outcome remains the same simply because the $R V$ associated with a patch has its sudden, sharp minimum and maximum at its appearance and disappearance, respectively.

\subsection{RV measurements of individual features}

If the observational schemes are designed to monitor the progress across the parent stellar line of blips caused by individual spots, the nature of the exercise is very different from that of measuring the $R V$ variations of the whole line.

A fundamental difference arises because of the logistics behind the data collection. It is obvious that measurements can only be obtained when a blip manifests itself, i.e., when the patch is apparent on the visible hemisphere of the star. No measurements are made when the patch is on the rear hemisphere

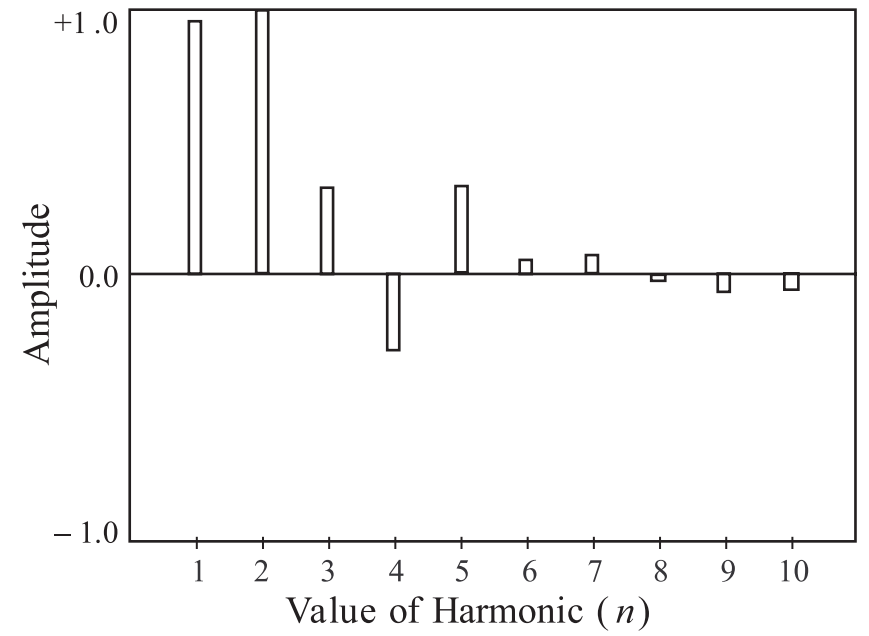

Fig. 7. The values of the amplitudes of the sine components associated with the harmonics of the expected variations based on $R V$ measurements of complete line profiles summarised by Eq. (9). The values have been normalised to that of the second harmonic, this having a slightly higher amplitude than the fundamental.

of the star. The records do not follow the patch over the full rotational cycle of the star, linking features which disappear on the red wing of the line profile to those which appear on the blue wing half a period later. Consequently, the sampling of the data is restricted. For photometry or whole line $R V$ studies, the data are assembled at random relative to the phase position of the patch and measurements are taken when the effect of the patch is not apparent. As a consequence, the outcomes of simple period analyses based on photometry or whole line $R V$ measurements relative to blip $R V$ monitoring are likely to be different.

For the purpose of describing the $R V$ spectral blip variation in the form of a Fourier series, the underlying periodic waveform of the behaviour may be expressed in the form

$R V(\phi)=V_{*}+V_{\mathrm{E}} \sin \phi / 2$,

as displayed in Fig. 8, with the periodic interval $-\pi$ to $+\pi$ being $\equiv P_{0} / 2$. It is obvious that the first harmonic corresponds to a period of $P_{0} / 2$ and that the underlying rotational period, $P_{0}$, is unlikely to show in any periodogram.

Analysis of Eq. (10) shows that its Fourier series comprises sine components only and may be expressed as

$\frac{-2 V_{\mathrm{E}}}{\pi} \sum_{n=1}^{\infty} \frac{n \cos n \pi \sin n \phi}{\left(n^{2}-\frac{1}{4}\right)}$

Again, as for Eq. (8), with the numerator carrying the factor $n$, many of the early harmonics carry significant power. To appreciate this, the amplitudes, $S_{n}$, are listed below together with the associated period relative to the underlying rotational period of the star, it being remembered that the 


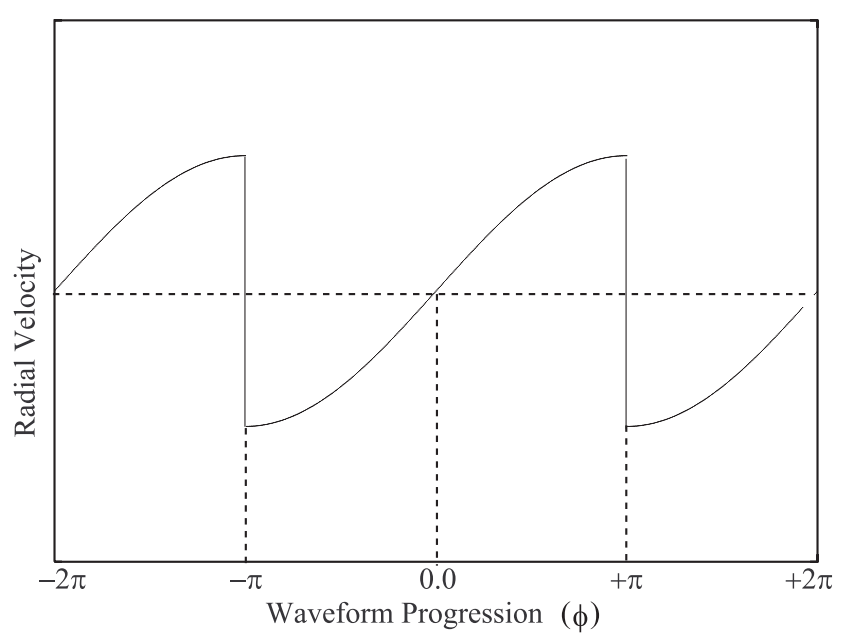

Fig. 8. The schematic behaviour of the radial velocity associated with a spot based on the notion that sampling only occurs when the spot is apparent on the visible hemisphere The horizontal axis $(\phi)$ is such that the interval $-\pi$ to $+\pi$ is equivalent to a time interval of $P_{0} / 2$.

harmonics are related to the apparent period, $P_{0} / 2$. Thus $S_{1}\left(P_{0} / 2\right) \propto \frac{8 V_{\mathrm{E}}}{3 \pi}, \quad S_{2}\left(P_{0} / 4\right) \propto-\frac{16 V_{\mathrm{E}}}{15 \pi}$,
$S_{3}\left(P_{0} / 6\right) \propto \frac{24 V_{\mathrm{E}}}{35 \pi}, \quad S_{4}\left(P_{0} / 8\right) \propto-\frac{32 V_{\mathrm{E}}}{63 \pi}$,
$S_{5}\left(P_{0} / 10\right) \propto \frac{40 V_{\mathrm{E}}}{99 \pi}, \quad S_{6}\left(P_{0} / 12\right) \propto-\frac{48 V_{\mathrm{E}}}{143 \pi}$,
$S_{7}\left(P_{0} / 14\right) \propto \frac{56 V_{\mathrm{E}}}{195 \pi}, \quad S_{8}\left(P_{0} / 16\right) \propto-\frac{64 V_{\mathrm{E}}}{255 \pi}$,
$S_{9}\left(P_{0} / 18\right) \propto \frac{144 V_{\mathrm{E}}}{323 \pi}, \quad S_{10}\left(P_{0} / 20\right) \propto-\frac{80 V_{\mathrm{E}}}{399 \pi}$, etc.

Although both even and odd harmonics are likely to show in the immediate periodogram, when translated to harmonics of the underlying rotational period, these correspond only to even ones.

\section{Polarimetric periodicities}

Any co-rotating patch disturbs the global radiation field from being spherically symmetric and consequently a polarization signal may be generated. There are many possible polarigenic mechanisms within stellar atmospheres that might give rise to measurable signals, varying according to the aspect and location of the patch, with periodicity linked to the rotation of the star. These include polarization related to magnetic patches (see, for example, the model by Landi Degl'Innocenti 1982), or by scattering from inhomogeneities in the distribution of atmospheric dust or free electrons (see, for example, the model of Brown \& McLean 1977)

Using the latter mechanism to provide an example for the behaviour of the signal, the degree of polarization generated by

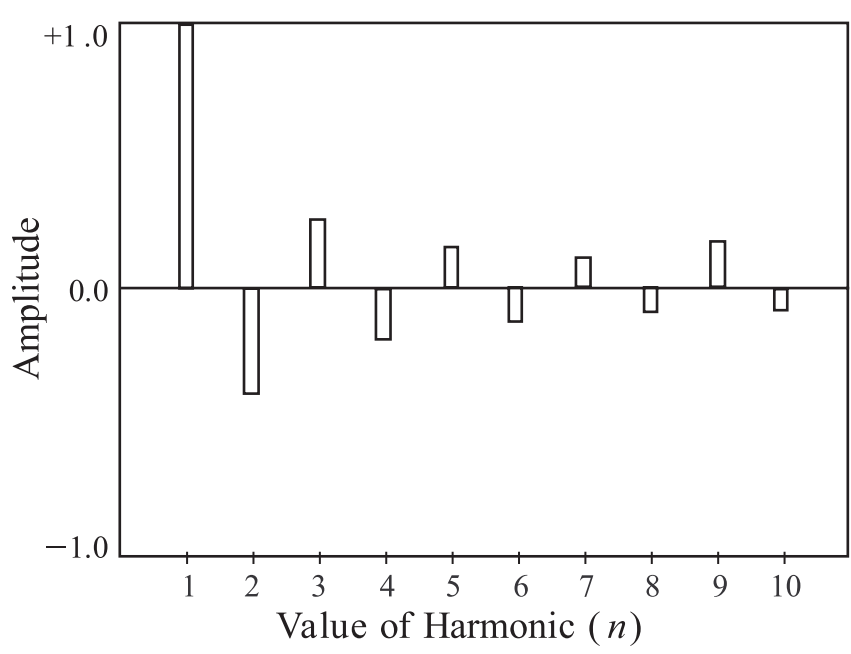

Fig. 9. The values of the amplitudes of the sine components associated with the harmonics of the expected variations based on $R V$ measurements of blips with stellar line profiles as summarised by Eq. (10). The values have been normalised to that of the first harmonic; it is to be noted that the period of the first harmonic corresponds to the half value of the rotational period.

a localized optically thin electron cloud illuminated by a point source may be written as

$p(\theta)=\frac{I_{\mathrm{p}}(\theta)}{I_{\mathrm{tot}}(\theta)}=\frac{\sin ^{2} \theta}{1+\cos ^{2} \theta}$,

where $I_{\mathrm{p}}$ is the polarized flux from the scattering, $I_{\text {tot }}$, the total flux and $\theta$ is the phase angle of the scattering (see, for example, Dolginov et al. 1995). For a globule of electrons close to the star, the value of $p$ would be smeared as the cloud illumination involves a range of scattering angles. In addition, the observed polarization would be very much diluted by the global radiation, $I_{*}$, received directly from the star. Hence the behaviour of the signal may be represented as

$p(\theta)=\frac{I_{\mathrm{p}}(\theta)}{I_{\mathrm{tot}}+I_{*}}$

Since $I_{*} \gg I_{\mathrm{tot}}$, the form of the polarimetric variation may be simplified to

$p(\theta)=p_{\mathrm{e}} \sin ^{2} \theta$,

with the magnitude of the signal, $p_{\mathrm{e}}$, depending on the number of electrons in the scattering cloud.

The form of the polarimetric behaviour of the radiation scattered by the cloud and that expected for the stellar measurements is depicted in Fig. 10. Analysis of Eq. (14) shows that its Fourier series comprises cosine components only which are summarised by

$\frac{p_{\mathrm{e}}}{\pi} \sum_{n=1}^{\infty}\left[\frac{\sin \left(\frac{n \pi}{2}\right)}{n}-\frac{\sin \left(\frac{(n+2) \pi}{2}\right)}{2(n+2)}-\frac{\sin \left(\frac{(n-2) \pi}{2}\right)}{2(n-2)}\right] \cos n \theta$.

By considering the contributions to the summation in turn, it is immediately apparent that all even harmonics have zero amplitude; the amplitudes, $C_{n}$, of the odd harmonics are calculated 


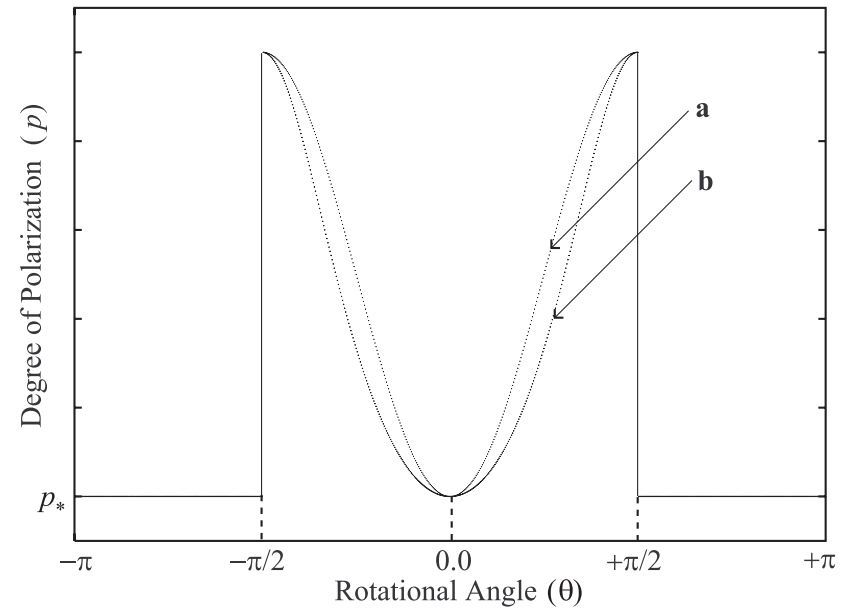

Fig. 10. The schematic behaviour of the degree of polarization, $p$, associated with a patch in co-rotation with the star, the signal generated by electron scattering. Curve a corresponds to the variation based on pure electron scattering, with $p=1.0$ at $\theta= \pm \pi / 2$. Curve $\mathbf{b}$ describes the expected measured behaviour with the direct radiation from the star diluting the signal (see Eq. (14)); the maximum value of $p$ depends on the number of electrons within the patch and the dilution factor. The signal may not reduce to zero at $\theta=0^{\circ}$ as there may be a constant contribution of $p_{*}$ from the interstellar medium.

from Eq. (15) as

$$
\begin{aligned}
& C_{1}\left(P_{0}\right)=+\frac{2 p_{\mathrm{e}}}{3 \pi}, \\
& C_{3}\left(P_{0} / 3\right)=-\frac{14 p_{\mathrm{e}}}{15 \pi}, \\
& C_{5}\left(P_{0} / 5\right)=+\frac{46 p_{\mathrm{e}}}{105 \pi}, \\
& C_{7}\left(P_{0} / 7\right)=-\frac{29 p_{\mathrm{e}}}{252 \pi}, \\
& C_{9}\left(P_{0} / 9\right)=+\frac{158 p_{\mathrm{e}}}{693 \pi}, \text { etc. }
\end{aligned}
$$

Based on these values, the expected form of any polarimetric periodogram is depicted in Fig. 11. It is interesting to note that the dominant component is the third harmonic and is more likely to be detected in any period search, rather than the fundamental of the stellar rotational period.

\section{Conclusion}

By taking a simple model involving a photospheric disturbance in co-rotation with a star, it is demonstrated that the immediate outcomes in terms of detection of periodicity significantly depend on the observational diagnostic technique that is used. Although the detailed strengths of the amplitudes appearing in any periodogram depend on the distribution of disturbing patches on the star, on effects associated with limb darkening, on the inclination of the stellar rotational pole, etc., a basic conclusion remains that there is high likelihood that integer submultiples of the stellar rotational period will be detected. Over and above the full astrophysical details associated with the generation of the signal, the distribution of power to these harmonics is very dependent on the observational diagnostic technique

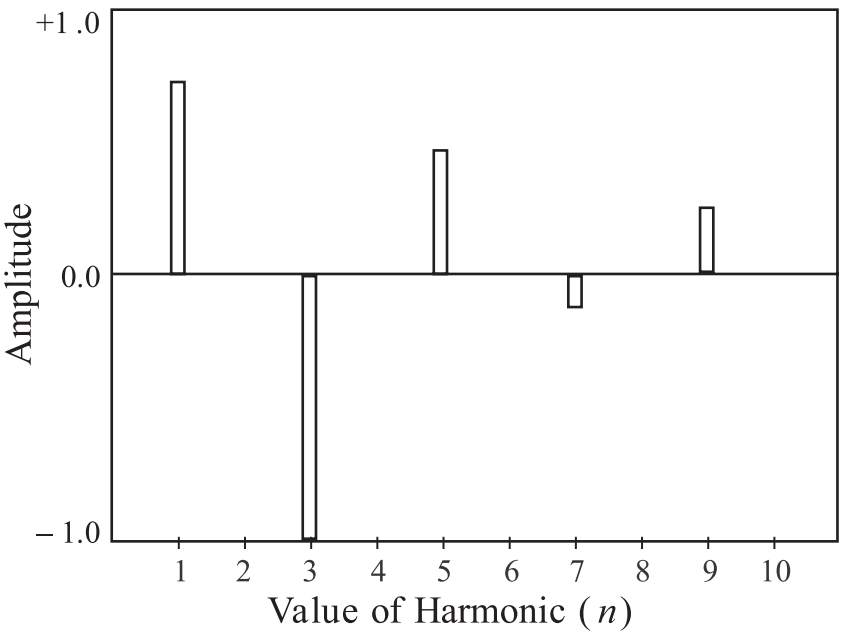

Fig. 11. The values of the amplitudes of the cosine components associated with the harmonics of the expected variations based on polarimetric measurements as summarised by Eq. (15). The values have been normalised to that of the third harmonic, this having a higher amplitude than the fundamental.

that is applied and on the subsequent data reduction process. It has been shown that data from regular photometry, line profile photometry, $R V$ measurements of line profiles, $R V$ measurements of individual disturbances within a line, and polarimetry would all provide periodograms exhibiting harmonics of the fundamental but with differing relative powers.

If photometry is undertaken, then, period analysis is likely to provide a value for the fundamental rotational period with some power also being present in the second harmonic.

For the case of $R V$ measurements, data values for complete line profiles are likely to provide the strongest periodicity corresponding to the second harmonic, with the fundamental also being strong if the measurement time windows are sufficiently wide. Relative to photometry, however, and, if the observational windows are relatively short in respect of the stellar rotational period, there is high likelihood that the third, fourth, fifth and higher harmonics will show, these being the integer submultiple values of $N=3,4,5$, etc. of the rotational period. For the physical interpretation, their appearance should be considered in terms of an integer relationship to a longer period.

If the data base relies on measurements of the $R V$ changes or "blips" appearing within the parent line, then the observational strategy dictates that the outcome is likely to provide a fundamental period of one half that of the rotational period. Higher harmonics are also very likely to show strongly, particularly for observational runs which are short relative to the underlying rotational period. These ensuing shorter periods correspond to integer submultiples values of $N=4,6,8$, etc. of the rotational period.

For polarimetric observations, it is the third harmonic of the rotational period that is most likely to be detected in any period analysis, with the fundamental and other odd harmonics also showing strongly. Again, care should be taken in promoting the strongest showing component as being the fundamental stellar rotational period. 
At a simplistic level, the analysis here appears to provide reason and support to Harmanec's (1999) thesis that the presence of integer submultiple periods in the behaviour of $\epsilon$ Per relates to the scenario of engendered signals by rotational modulation. In a paper cited by Harmanec as evidence for the presence of such integer submultiple periods, Gies \& Kullavanijaya (1988) detected four periods by monitoring the intensities of line cores. They discussed a possible origin in terms of obscurating orbiting material but comment that the presence of four periodicities would require the existence of a very arbitrary set of four different structures in the circumstellar material and thus the variations could not be due to periodic obscuration by circumstellar clouds. The harmonic analysis here shows that the four periods could be engendered simply by the behaviour of the signal produced by a single obscurating patch in rotation about the star, without the need to invoke the presence of some particular, more complicated, geometry. As it has since turned out, however, the situation with respect of $\epsilon$ Per has become more complex by the detection by Gies et al. (1999) of additional periods interspersed between the original four used as evidence to promote the integer submultiple theory.

More importantly, however, the detection of integer submultiple periods does not in itself automatically support the notion of their production by rotational modulation. Any kind of cyclic phenomenon, including non-radial pulsations, which produces non-sinusoidal variations with forms dependent on the measurement parameter will give rise to periodograms containing harmonics of the fundamental period. Again, according to how the disturbance affects the signals associated with photometry, spectrophotometry, radial velocities or polarimetry, the various detected harmonics will have powers according to the form of the non-sinusoidal behaviour of the selected measurement parameter.

In discriminating between different basic underlying physical causes of the variability, e.g., rotational modulation or non-radial pulsations, it might be considered that simultaneous measurements involving the various measurement diagnostics might be undertaken. From comparison of the expected relative strengths of the harmonics associated with each measurement parameter and the observed powers in the periodograms, it might be surmised that the issue of the preferred model could be resolved. Unfortunately, the results are unlikely to be conclusive as, for example, in the spot model discussed above, the powers of the various harmonics depend on the spot distribution over the stellar surface and this could be adjusted by ad hoc geometries.

A further general conclusion is that if period searches are undertaken to explore any astrophysical scenario, immediate outcomes may give rises to confusions depending on the particular observational diagnostic that is applied. As for the model discussed above, for example, the harmonic providing the most power in the recorded signal is significantly different depending on whether photometry or polarimetry is used to detect the fundamental rotational period. Care and thought must be practised in presenting the outcomes from and interpretations of the various measurement diagnostics.

Acknowledgements. The author wishes to thank the anonymous referee for guidance on some of the issues in the paper.

\section{References}

Brown, J. C., \& McLean, I. S. 1977, A\&A, 57, 141

Dolginov, A. Z., Gnedin, Yu. N., \& Silant'ev, N. A. 1995, Propagation and Polarization in Cosmic Media (Basel, Switzerland: Gordon \& Breach)

Gies, D. R., \& Kullavanijaya, A. 1988, ApJ, 326, 813

Gies, D. R., Kambe, E., Josephs, T. S., et al. 1999, ApJ, 525, 420

Harmanec, P. 1999, A\&A, 341, 867

Harmanec, P. 1989, Bull. Astron. Inst. Czechosl., 40, 201

Harmanec, P., \& Tarasov, A. E. 1990, Bull. Astron. Inst. Czechosl., 41,273

Harmanec, P. 1987, Inf. Bull. Var. Stars No. 3097

James, J. F. 2002, A Student's Guide to Fourier Transforms - 2nd ed. (Cambridge Univ. Press)

Landi Degl'Innocenti, E. 1982, A\&A, 110, 25

Van Leeuwen, F., Alphenaar, P., \& Meys, J. M. M. 1987, A\&AS, 67, 483

Rivinius, Th., Baade, D., Štefl, S., et al. 1998, A\&A, 336, 177

Roberts, D. H., Lehár, J., \& Dreher, J. W. 1987, AJ, 93, 968

Smith, M. A. 1985, ApJ, 288, 266

Smith, M. A., Fullerton, A. W., \& Percy, J. R. 1987, ApJ, 320, 768

Vogt, S. S., \& Penrod, G. D. 1983, ApJ, 275, 661 